\title{
LEAST PERIODS OF FACTORS OF INFINITE WORDS
}

\author{
JAmes D. CURRIE ${ }^{1}$ AND KAlle SAARI ${ }^{2}$
}

\begin{abstract}
We show that any positive integer is the least period of a factor of the Thue-Morse word. We also characterize the set of least periods of factors of a Sturmian word. In particular, the corresponding set for the Fibonacci word is the set of Fibonacci numbers. As a byproduct of our results, we give several new proofs and tightenings of well-known properties of Sturmian words.
\end{abstract}

Mathematics Subject Classification. 68R15.

\section{INTRODUCTION}

The combinatorial study of infinite words often entails considering periods of factors. For example, showing that an infinite word has a bounded critical exponent requires showing, perhaps implicitly, that the ratio between a factor and its least period is bounded. Therefore it seems natural to study directly the set of least periods of factors of an infinite word; we call this set the period set of an infinite word. To our knowledge, this is a novel area of inquiry into the periodicity of finite and infinite words [11], Chapter 8 .

This paper initiates the study of the period set of infinite words. It is easy to see that the period set of an infinite word is finite if and only if the word is purely periodic. Therefore infinite aperiodic words give rise to infinite period sets, and it is natural to ask what kind of restrictions period sets have to obey. It is plain that the period set of an aperiodic infinite word must include periods 1, 2, and 3 . But already 4 is avoidable, as is witnessed by the Fibonacci word, see Corollary 4 .

\footnotetext{
Keywords and phrases. Periodicity, Fibonacci word, Thue-Morse word, Sturmian word.

* Work of the first author supported by a Discovery Grant from NSERC.

** Work of the second author supported by the Finnish Academy under grant 8206039.

1 Department of Mathematics \& Statistics, University of Winnipeg, Winnipeg, R3B2E9, Canada

2 Department of Mathematics and Turku Centre for Computer Science, University of Turku, Turku, Finland; kasaar@utu.fi
} 
In this paper we will characterize the period sets of the Thue-Morse word and of all Sturmian words. These much studied words have applications and connections to several fields, such as algebra, number theory, ergodic theory, crystallography, computer graphics, and text algorithms; see [1] and [11], Chapter 2 and the references therein. The characterizations of the period sets show that the gaps in the period set of the Fibonacci word grow exponentially, while the gaps in the period set of the Thue-Morse word have the lowest possible growth an aperiodic infinite word can have. As a by-product of our work, we give new proofs, tightenings, and generalizations of some known properties of Sturmian words.

An outline of this paper is as follows: in Section 2, we set the terminology used in the paper, and mention some basic results. In Section 3, we show that any positive integer is the least period of some factor of the Thue-Morse word. In Section 4, we characterize the set of least periods of a Sturmian word. Finally, in Section 5, we give four applications of our results, including a tightening of a result by de Luca and De Luca [7] and a characterization of the least periods of standard words.

\section{Preliminaries}

In this section we briefly define the terminology used in this work. For a statement without a citation in this section, we refer to $[4,10,11]$.

We will be dealing with words over the alphabet $\{0,1\}$. The set of all such words, including the empty word, is denoted by $\{0,1\}^{*}$.

Let $w=a_{1} a_{2} \cdots a_{n}$ be a word with $a_{i} \in\{0,1\}$ and $n \geq 1$. The length of $w$ is the integer $n$, and is denoted by $|w|$. We denote the number of occurrences of a letter $a \in\{0,1\}$ in $w$ by $|w|_{a}$.

A factor of $w$ is a word of the form $u=a_{i} a_{i+1} \cdots a_{k}$ with $1 \leq i \leq k \leq n$. It is a prefix if $i=1$ and a suffix if $k=n$. In each case, we add the attribute proper if $w \neq u$.

Let $0 \leq i<|w|$. The word $a_{i+1} a_{i+2} a_{i+3} \cdots a_{n} a_{1} a_{2} \cdots a_{i}$ is called a conjugate of $w$, and is denoted by $\sigma^{i}(w)$.

We write $w^{\prime}=a_{1} a_{2} \cdots a_{n-1}$ and $\sim w=a_{2} a_{3} \cdots a_{n}$. The reverse of $w$ is the word $a_{n} a_{n-1} \cdots a_{1}$, and we denote it by $w^{R}$. We denote by $\bar{w}$ the word obtained from $w$ by exchanging 0 's and 1's; it is called the complement of $w$.

A period of the word $w$ is an integer $p \geq 1$ such that, for all $i=1,2, \ldots, n-p$, we have $a_{i}=a_{i+p}$. The word $w$ is said to be a rational power of the word $u=a_{1} a_{2} \cdots a_{p}$, and $u$ is called a word period of $w$. If no period $p$ divides the length of $w$, then $w$ is termed primitive. Primitivity of a word implies that all conjugates of the word are distinct.

In this work, we are interested in the least period of a word $w$, which we denote by $p(w)$. The word $w$ is called unbordered if $p(w)=|w|$. Finally, the prefix of $w$ of length $p(w)$ is called the fractional root of $w$.

Let the words in $\{0,1\}^{*}$ be ordered by the lexicographic order induced by the relation $0<1$. If $w$ is a primitive word, then its least conjugate with respect to 
the lexicographic order is called a Lyndon word. If $|w|>1$, we get a different Lyndon word by using the lexicographic order induced by the relation $1<0$. One of the basic properties of a Lyndon word is that it is unbordered.

Let $\mathbf{x}$ be an infinite word, that is, a mapping from the nonnegative integers to a finite alphabet. The notion of a factor is extended naturally to infinite words with the agreement that a factor is always a finite word. The set of finite factors of $\mathbf{x}$ is denoted by $F(\mathbf{x})$. We call the set of least periods of all factors of $\mathbf{x}$ the period set of $\mathbf{x}$.

A morphism is a mapping $h:\{0,1\}^{*} \rightarrow\{0,1\}^{*}$ with the property that $h(u v)=$ $h(u) h(v)$ for every $u, v \in\{0,1\}^{*}$. The domain of $h$ extends to infinite words such that if

$$
\mathbf{x}=a_{1} a_{2} \cdots a_{n} \cdots, \quad \text { then } h(\mathbf{x})=h\left(a_{1}\right) h\left(a_{2}\right) \cdots h\left(a_{n}\right) \cdots
$$

The Thue-Morse word, denoted by $\mathbf{t}$, is the infinite word starting with the letter 0 that is a fixed point of the morphism $\mu:\{0,1\}^{*} \rightarrow\{0,1\}^{*}$ determined by $\mu: 0 \mapsto$ $01,1 \mapsto 10$. If $u$ is a factor of $\mathbf{t}$, then so are $\bar{u}$ and $u^{R}$. The Thue-Morse word is overlap-free, which means that $\mathbf{t}$ does not have a factor of the form $u u a$, where $u$ is a nonempty word and $a$ is the first letter of $u$.

A Sturmian word is an infinite word $\mathbf{x}$ over $\{0,1\}$ such that, for every integer $n \geq 1$, the word $\mathrm{x}$ has precisely $n+1$ different factors of length $n$. The frequency of letters 0 and 1 in $\mathbf{x}$ exists; the frequency of 1 is called the slope of $\mathbf{x}$, and we denote it by $\theta$. The slope $\theta$ is an irrational number, and therefore it has an infinite continued fraction expansion

$$
\theta=\left[0, d_{1}+1, d_{2}, d_{3}, \ldots\right]
$$

where $d_{1} \geq 0$ and $d_{n} \geq 1$ for $n \geq 2$.

Next we define words $s_{n}$ corresponding to the expansion (1) as follows:

$$
s_{-1}=1, \quad s_{0}=0, \quad s_{n}=s_{n-1}^{d_{n}} s_{n-2} \quad(n \geq 1) .
$$

Words that can be recursively defined as above are called standard. All standard words are primitive. Furthermore, consecutive standard words $s_{n-1}$ and $s_{n}$ are near-commutative in the following sense: if $n \geq 1$, then there exists a word $p_{n}$ such that

$$
s_{n} s_{n-1}=p_{n} a \bar{a} \quad \text { and } \quad s_{n-1} s_{n}=p_{n} \bar{a} a,
$$

where $a \in\{0,1\}$. Therefore, for $n \geq 2$, we have

$$
s_{n} s_{n-1}^{\prime \prime}=s_{n-1} s_{n}^{\prime \prime}
$$

Let us denote $q_{n}=\left|s_{n}\right|$ for all $n \geq-1$.

The standard words corresponding to the slope $\theta$ are related to $\mathbf{x}$ in the following way. Since $s_{n}$ is a prefix of $s_{n+1}$ for all $n \geq 1$, there is a unique infinite word, which we denote by $\mathbf{c}$, such that $s_{n}$ is a prefix of $\mathbf{c}$ for all $n \geq 1$. The word $\mathbf{c}$ is called the characteristic word with slope $\theta$. The sets of finite factors of $\mathbf{x}$ and $\mathbf{c}$ coincide, that is, we have $F(\mathbf{c})=F(\mathbf{x})$. 
Since $\mathbf{x}$ has $n+1$ factors of length $n$, it follows that there exist precisely one factor $u$ of length $n$ such that both $0 u$ and $1 u$ are factors of $\mathbf{x}$. Such a factor is called left special. A factor of $\mathbf{x}$ is left special if and only if it is a prefix of $\mathbf{c}$.

The set of factors of $\mathbf{x}$ is closed under reversal, that is to say, if $u \in F(\mathbf{x})$, then also $u^{R} \in F(\mathbf{x})$.

Now we will adopt a notation from [13]. For each integer $n \geq 1$, there exists a unique representation

$$
n=d_{1}+d_{2}+\cdots+d_{i-1}+j, \quad 1 \leq j \leq d_{i} .
$$

With this representation, we denote

$$
t_{n}=s_{i-1}^{j} s_{i-2} .
$$

It is also useful to denote $t_{-1}=1$ and $t_{0}=0$. Observe that $t_{d_{1}+\cdots+d_{n}}=s_{n}$ for all $n \geq 1$.

The following result by Berstel [2] is one of the key observations we need in characterizing the period set of a Sturmian word.

Theorem 1 (Berstel). For $n \geq 2$, the longest prefix of $\mathbf{c}$ that is a rational power of the word $s_{n}$ is $s_{n}^{d_{n+1}+1} s_{n-1}^{\prime \prime}$.

\section{Periods of Factors of the Thue-Morse Word}

In this section we will show that every positive integer is the least period of some factor of the Thue-Morse word. To do that, we need some auxiliary results. Recall that $\mu$ denotes the morphism given by $\mu: 0 \mapsto 01,1 \mapsto 10$.

Lemma 1. Let $u$ be a factor of the Thue-Morse word $\mathbf{t}$. Then $u$ does not have any odd period $p$ such that $p<|u|-3$.

Proof. Suppose that $u$ has an odd period $p$ with $p<|u|-3$. We may suppose that $p \geq 3$ because $\mathbf{t}$ does not contain 000 or 111 . Then $|u| \geq 7$. Let $v$ be the prefix of $u$ of (odd) length $p+4$.

Observe that, since $v$ is a factor of $\mathbf{t}$, also $v^{R}$ and $\bar{v}$ are factors of $\mathbf{t}$. Therefore, without loss of generality, replacing $v$ by its reversal or complement or both if necessary, write $v=\mu(w) a=v_{0} v_{1} v_{2} \cdots v_{p+3}$, where $v_{i}, a \in\{0,1\}$, and $v_{0}=0$.

Since $v$ has period $p$, we find that $v_{p}=v_{0}=0$, so that $v_{p-1} v_{p}=10$. Similarly, $v_{p+1}=v_{1}=1$, so that $v_{p+1} v_{p+2}=10$. Thus $v_{2}=v_{p+2}=0$, whence $v_{2} v_{3}=01$. This implies that $v_{p+3}=v_{3}=1$, and $v$ contains the overlap $v_{p-1} v_{p} v_{p+1} v_{p+2} v_{p+3}=$ 10101, which is impossible because $\mathbf{t}$ is overlap-free.

Recall that we denote by $\sim u, u^{\prime}$ and $\sim u^{\prime}$ the words obtained from $u$ by deleting respectively the first letter, the last letter, or the first and last letters.

Lemma 2. Let $u=\mu(w)$, some $w \in\{0,1\}^{+}$. Let $v=\sim u^{\prime}$. Suppose that $v$ has an even period $2 r<|v|$. Then $w$ has period $r$. 
Proof. Write $w=w_{0} w_{1} w_{2} \cdots w_{s-1} w_{s}$ and $v=v_{0} v_{1} v_{2} \cdots v_{2 s-1}$, so that $r<s$. We see that

$$
v=\bar{w}_{0} w_{1} \bar{w}_{1} w_{2} \bar{w}_{2} \cdots w_{s-1} \bar{w}_{s-1} w_{s} .
$$

Since $v$ has period $2 r$, we have $\bar{w}_{i}=v_{2 i}=v_{2 i+2 r}=\bar{w}_{i+r}$ whenever $0 \leq 2 i+2 r \leq$ $|v|-2$, that is, $0 \leq i \leq s-1-r$. Therefore,

$$
w_{i}=w_{i+r} \quad \text { for all } 0 \leq i \leq s-1-r
$$

Similarly, since $v$ has period $2 r$, we have $w_{i}=v_{2 i-1}=v_{2 i-1+2 r}=w_{i+r}$ whenever $0 \leq 2 i-1+2 r \leq|v|-1$, that is, $1 \leq i \leq s-r$. In total,

$$
w_{i}=w_{i+r} \quad \text { for all } 0 \leq i \leq s-r .
$$

The claim in the lemma above does not hold if we allow $|v|=2 r$. Indeed, if $w=01$, then $v=11$. Even though 2 is plainly a period of $v$, the word $w$ certainly does not have period 1 .

Corollary 1. Let $u=\mu(w)$, some $w \in\{0,1\}^{+}$. Let $v$ be obtained from $u$ by possibly deleting first or last or both letters; that is, let $v$ be one of $u, u^{\prime}, \sim u, \sim u^{\prime}$. Then $v$ has period $2 r<|u|-2$ if and only if $w$ has period $r$.

Proof. Suppose that $v$ has period $2 r$. Then $\sim u^{\prime}$ is a factor of $v$ and has period $2 r$, so that, by Lemma 2, $w$ has period $r$.

If $w$ has period $r$, then $\mu(w)$ has period $2 r$ since $|\mu(0)|=|\mu(1)|=2$. It follows that the factor $v$ of $\mu(w)$ has period $2 r$.

Lemma 3. Let $r \geq 4$ be a positive integer. Then the following statements hold:

(i) if $r \equiv 4(\bmod 6)$, then $\mathbf{t}$ has a factor $u$ of the form $u=00 y 11$ with $|u|=r$ and $p(u)=r$;

(ii) if $r \equiv 0,2,3$, or $5(\bmod 6)$, then $\mathbf{t}$ has a factor $u$ of the form $u=00 y 101$ with $|u|=r$ and $p(u)=r$;

(iii) if $r \equiv 0,1$, or $3(\bmod 6)$, then $\mathbf{t}$ has a factor $u$ of the form $u=00 y 010$ with $|u|=r+1$ and $p(u)=r$.

Proof. We prove this by induction. The item (i) with $r=4$ is witnessed by the factor 0011. The item (ii) with $r=5,6,8$, or 9 is witnessed by factors

$$
\text { 00101, 001101, 00101101, and } 001100101 .
$$

The item (iii) with $r=6,7,9$ is witnessed by factors

$$
\text { 0011010, 00110010, and 0011010010. }
$$

Let us now assume that $r \geq 10$, and that the lemma is satisfied for all smaller values of $r$.

Case 1. $r \equiv 0(\bmod 6)$. First, let $s=r / 2$. Then either $s \equiv 0$, or $3(\bmod 6)$, and $s<r$. By the minimality of $r$ and the item (iii), there is a factor $w$ of $\mathbf{t}$ of the form 
$00 z 010$ having length $s+1$ and least period $s$. Let $u=\sim \mu\left(\bar{w}^{R}\right)$. Then $u$ is a factor of $\mathbf{t}$, it is of length $r+1$, and it has the form $u=00 y 010$, where $y=110 \mu\left(\bar{z}^{R}\right) 1$.

Evidently, $u$ has period $r$. Corollary 1 implies that $u$ has no even period shorter than $r=2 s$. Writing $u=u_{0} u_{1} u_{2} \cdots u_{r}$, we see that $u_{0} \neq u_{r-1}, u_{2} \neq u_{r}, u_{3} \neq u_{r}$, showing that $u$ does not have period $r-1, r-2$, or $r-3$. By Lemma 1, $u$ can have no odd period, and therefore the least period of $u$ is $r$, witnessing the item (iii).

Next, let $v=\sim \mu\left(\bar{w}^{R}\right)^{\prime}$. Then $v$ is of length $r$, and thus has period $r$. Furthermore, $v=\sim \mu\left(101 \bar{z}^{R} 11\right)^{\prime}$ has the form $00 y 101$, where $y=110 \mu\left(\bar{z}^{R}\right)$. It has no even period shorter than $r=2 s$ by Corollary 1 . Writing $v=v_{0} v_{1} v_{2} \cdots v_{r-1}$, we see that $v_{0} \neq v_{r-1}, v_{1} \neq v_{r-1}, v_{0} \neq v_{r-3}$, showing that $v$ does not have period $r-1$, $r-2$, or $r-3$. By Lemma 1, $v$ can have no odd period. Thus the least period of $v$ is $r$, witnessing the item (ii).

Case 2. $r \equiv 3(\bmod 6)$. First, let $s=(r+3) / 2$. Then either $s \equiv 0$, or $3(\bmod 6)$, and $s<r$. Thus there is a factor $w$ of $\mathbf{t}$ of the form 00z101 having length $s$ and least period $s$. Let $u=\sim \mu\left(w^{R}\right)^{\prime}$. Then $u$ is a factor of $\mathbf{t}$, it is of length $r+1$, and it is of the form $u=00 y 010$, where $y=110 \mu\left(z^{R}\right)$.

Evidently, the word $u$ has period $r$. Corollary 1 implies that it has no even period strictly shorter than $|u|=r+1=2 s-2$. Writing $u=u_{0} u_{1} u_{2} \cdots u_{r}$, we see that $u_{0} \neq u_{r-1}, u_{1} \neq u_{r-1}, u_{3} \neq u_{r}$, showing that $u$ does not have period $r-1$, $r-2$, or $r-3$. By Lemma 1, $u$ can have no odd period less than $r$. Thus the least period of $u$ is $r$, witnessing the item (iii).

Next, let $s=(r+1) / 2$. Then either $s \equiv 2$, or $5(\bmod 6)$. There is a factor $v$ of $\mathbf{t}$ of the form $00 z 101$ having length $s$ and least period $s$. Let $u=\sim \mu\left(v^{R}\right)$. Then $u$ is a factor of $\mathbf{t}$, it is of length $r$, and it has the form $u=00 y 101$, where $y=110 \mu\left(z^{R}\right) 0$.

Evidently, the word $u$ has period $|u|=r$. Corollary 1 implies that it has no even period strictly shorter than $r+1=2 s$. Writing $u=u_{0} u_{1} u_{2} \ldots u_{r-1}$, we see that $u_{0} \neq u_{r-1}, u_{1} \neq u_{r-1}, u_{0} \neq u_{r-3}$, showing that $u$ does not have period $r-1$, $r-2$, or $r-3$. By Lemma 1, $u$ can have no odd period less than $r$. Thus the least period of $u$ is $r$, witnessing the item (ii).

Case 3. $r \equiv 1(\bmod 6)$. Let $s=(r+3) / 2$. Then either $s \equiv 2$, or $5(\bmod 6)$, and $s<r$. Thus there is a factor $v$ of $\mathbf{t}$ of the form $00 z 101$ having length $s$ and least period $s$. Let $u=\sim \mu\left(v^{R}\right)^{\prime}$. As in the previous case, $u$ is a factor of $\mathbf{t}$, it is of length $r+1$, has the form $u=00 y 010$, and its least period equals $r=|u|-1$, witnessing the item (iii).

Case 4. $r \equiv 4(\bmod 6)$. Let $s=r / 2$. Then either $s \equiv 2$, or $5(\bmod 6)$. There is a factor $v$ of $\mathbf{t}$ of the form $00 z 101$ having length $s$ and least period $s$. The word $v=00 z 101$ must be obtained by deleting the first and possibly last letter of some word $\mu(t)$, where $t$ is some factor of $\mathbf{t}$. Let $u$ denote a word of the form $u=1001 x 101$ that is obtained from $\mu(t)$ by possibly deleting the last letter.

Next we will show that $u$ has no even period less than $s$. To derive a contradiction, suppose that $u$ has period $2 k<s$. Then by Corollary 1 , the word $t$ has period $k$. But then, again by Corollary 1 , the word $v$ has a period $2 k<s$, a contradiction. 
Writing $u=u_{0} u_{1} \cdots u_{s}$, we see that $u_{1} \neq u_{s}, u_{2} \neq u_{s}, u_{1} \neq u_{s-2}$, so that $u$ does not have period $s-1, s-2$, or $s-3$. Therefore $u$ has no odd period less than $s$, and it follows that its least period is $s$.

We now let $w=\sim \mu(u)^{\prime}=00 y 11$, where $y=10110 \mu(x) 100$. Then $w$ is of length $r$. The same argument used before shows that $w$ has no even period less than $r$. Writing $w=w_{0} w_{1} \cdots w_{r-1}$, we see that $w_{0} \neq w_{r-1}, w_{0} \neq w_{r-2}, w_{1} \neq$ $w_{r-2}$, and so $w$ has no odd period less than $r$ either. Therefore the least period of $w$ is $r$, witnessing the item (i), as desired.

Case 5. $r \equiv 2(\bmod 6)$. Let $s=r / 2$. Then we have two possibilities.

If $s \equiv 1(\bmod 6)$, then $\mathbf{t}$ has a factor $w=00 z 010$ of length $s+1$, minimum period $s$. Let $v=\sim \mu\left(\bar{w}^{R}\right)^{\prime}$. Then $v$ has form $00 y 101$ with length $r$ and least period $r$, as can be seen as above.

If $s \equiv 4(\bmod 6)$, then $\mathbf{t}$ has a factor of the form $00 z 11$ having length $s$ and least period $s$. It follows that $u=100 z 11$ is a factor of $\mathbf{t}$ having length $s+1$ and its least period is $s$. Let $w=\sim \mu(u)^{\prime}$. Then $w=00 y 101$, where $y=101 \mu(z)$. As in previous cases, the word $w$ is of length $r$, and its least period is $r$, witnessing the item (ii).

Case 6. $r \equiv 5(\bmod 6)$. Let $s=(r+1) / 2$. Then either $s \equiv 0$, or $3(\bmod 6)$, and $s<r$. Therefore, $\mathbf{t}$ has a factor of the form $v=00 z 101$ having length $s$ and least period $s$. It follows that $u=\sim \mu\left(v^{R}\right)$ has the form $u=00 y 101$ where $y=110 \mu\left(z^{R}\right) 0$. As in previous cases, $u$ is of the length $r$, and the least period of $u$ is $r$, witnessing the item (ii).

Remark 1. The previous lemma shows that the Thue-Morse word has an unbordered factor for each length $r \not \equiv 1(\bmod 6)$. It is readily verified that all factors of length 7 are bordered. Since the factors of length 1 are trivially unbordered, it is natural to ask, for which lengths $r \equiv 1(\bmod 6)$ are all factors of length $r$ bordered. This question remains open.

We are ready for the main theorem of this section.

Theorem 2. For each integer $n \geq 1$, the Thue-Morse word has a factor of least period $n$.

Proof. The least periods $1,2,3$ are displayed by factors 0,01 , and 001 . For integers $n \geq 4$, appropriate factors exist according to Lemma 3 .

\section{Periods of Factors of Sturmian words}

In this section we will characterize the period sets of all Sturmian words, and by doing so, we obtain a few older results on Sturmian words as a by-product in the next section.

Let $\mathbf{x}$ be a Sturmian word with slope $\theta$. Denote the continued fraction expansion of $\theta$ by

$$
\theta=\left[0, d_{1}+1, d_{2}, d_{3}, \ldots\right] .
$$


Let $\left(s_{n}\right)_{n \geq-1}$ be the corresponding sequence of standard words, and let $\left(t_{m}\right)_{m \geq-1}$ denote the corresponding auxiliary words defined in (4). Further, let $\mathbf{c}$ denote the characteristic sequence with slope $\theta$. Observe that $d_{1} \geq 0$ and $d_{n} \geq 1$ for all $n \geq 2$. Since the period set of a sequence does not depend on the naming of letters, we may assume that $\mathbf{c}$ begins with 0 . Therefore, we assume in the rest of this section that $d_{1} \geq 1$.

Lemma 4. For $n \geq 0$, the word $s_{n}^{2}$ is a factor of $\mathbf{x}$. For $m \geq d_{1}$, the word $t_{m}^{2}$ is a factor of $\mathbf{x}$.

Proof. The word $s_{n+1}^{d_{n+2}} s_{n} s_{n+1}$ is a prefix of $s_{n+3}$, and therefore a factor of $\mathbf{x}$. Since $n \geq 0$ (and $d_{1} \geq 1$ ), the word $s_{n}$ is a prefix of $s_{n+1}$. Consequently, $s_{n}^{2}$ is a factor of $\mathbf{x}$.

If $m=d_{1}$, then $t_{m}^{2}=s_{1}^{2}$ occurs in $\mathbf{x}$. So, we may suppose that $m>d_{1}$. Then we have $t_{m}=s_{n}^{i} s_{n-1}$ for some integers $n \geq 1$ and $1 \leq i \leq d_{n+1}$. Since $s_{n+1}^{2}$ occurs in $\mathbf{x}$, we see that the word $s_{n}^{i} s_{n-1} s_{n}^{d_{n+1}} s_{n-1}$ occurs in $\mathbf{x}$. Since $n \geq 1$, the word $s_{n-1}$ is a prefix of $s_{n}$, and hence it follows that the square of the word $t_{m}=s_{n}^{i} s_{n-1}$ occurs in $\mathbf{x}$.

Corollary 2. For $m \geq-1$, all conjugates of $t_{m}$ are factors of $\mathbf{x}$.

Proof. The claim is trivial if $m$ equals -1 or 0 . When $1 \leq m<d_{1}$, the claim is witnessed by $s_{1}^{2}=0^{d_{1}} 10^{d_{1}} 1$. When $m \geq d_{1}$, the word $t_{m}^{2}$ occurs in $\mathbf{x}$, and so the claim obviously holds then as well.

The words $t_{m}$ clearly are standard, and hence primitive. Therefore all the conjugates of $t_{m}$ are distinct. Since all conjugates of $t_{m}$ are factors of $\mathbf{x}$, and $\mathbf{x}$ has $\left|t_{m}\right|+1$ factors of length $\left|t_{m}\right|$, it follows that $\mathbf{x}$ has precisely one factor of length $\left|t_{m}\right|$ that is not a conjugate of $t_{m}$. We call this factor the singular factor of $\mathbf{x}$ corresponding to $t_{m}{ }^{1}$. With this definition, $t_{-1}=1$ is the singular factor corresponding to $t_{0}=0$, and vice versa. We give the other singular factors in the next lemma.

Lemma 5. Let $m \geq 1$, and let a denote the last letter of $t_{m}=s_{n}^{i} s_{n-1}$. The singular factor corresponding to $t_{m}$ equals $\bar{a} t_{m}^{\prime}$, and it is bordered with period $q_{n}$.

Proof. First, observe that $n \geq 0$ and $1 \leq i \leq d_{n+1}$. It is clear that $s_{n+2} s_{n+1}$ is a prefix of $\mathbf{c}$, and hence a factor of $\mathbf{x}$. Since

$$
s_{n+2} s_{n+1}=s_{n+1}^{d_{n+2}} s_{n}^{d_{n+1}+1} s_{n-1},
$$

we see that the word $s_{n}^{i+1} s_{n-1}$ is a factor of $\mathbf{x}$.

First, suppose that $n=0$. Then the word $0^{i+1}$ occurs in $\mathbf{x}$, and it clearly is the singular factor corresponding to $s_{n}^{i} s_{n-1}=0^{i} 1$. The claim holds for $0^{i+1}$.

\footnotetext{
${ }^{1}$ Singular factors for Sturmian words seem to have been introduced by Cao and Wen [3], but only in cases that correspond to the words $s_{n}$.
} 
Next, suppose that $n=1$. Then

$$
s_{1}^{i+1} s_{0}=0^{d_{1}} 1\left(0^{d_{1}} 1\right)^{i} 0,
$$

and hence the word $1\left(0^{d_{1}} 1\right)^{i}$ occurs in $\mathbf{x}$, and it clearly is the singular factor corresponding to $s_{1}^{i} s_{0}$, satisfying the claim.

Finally, suppose that $n \geq 2$. Let us denote $s_{n}=s_{n}^{\prime \prime} a b$ and $s_{n-1}=s_{n-1}^{\prime \prime} b a$, where $a b \in\{01,10\}$. Equation (6) shows that the word $w=b s_{n}^{i} s_{n-1}^{\prime \prime} b$ is a factor of $\mathbf{x}$. Also, $w$ is not a conjugate of $s_{n}^{i} s_{n-1}$ because $|w|_{b}=\left|s_{n}^{i} s_{n-1}\right|_{b}+1$. Hence $w$ is the corresponding singular factor of $t_{m}$. Since $b=\bar{a}$, we have $w=\bar{a} t_{m}^{\prime}$. Furthermore, the word $w$ is bordered with period $q_{n}$ because

$$
w=b s_{n}^{i} s_{n-1}^{\prime \prime} b=\left(b s_{n}^{\prime \prime} a\right)^{i} b s_{n-1}^{\prime \prime} b,
$$

and $b s_{n-1}^{\prime \prime} b$ is a prefix of $b s_{n}^{\prime \prime} a$.

Lemma 6. Let $n \geq 0$ and $i \geq 1$. Denote $w_{j}=\sigma^{j}\left(s_{n}^{i} s_{n-1}\right)$. Then $w_{j}$ has a period

$$
\begin{cases}q_{n} & \text { if } 0 \leq j \leq q_{n}-2 ; \\ (i-1) q_{n}+q_{n-1} & \text { if } q_{n} \leq j \leq i q_{n}+q_{n-1}-2 .\end{cases}
$$

Furthermore, $w_{j}$ is unbordered if and only if $j=q_{n}-1$ or $j=i q_{n}+q_{n-1}-1$, and then $w_{j}$ is a Lyndon word.

Proof. The claim is readily verified for $n=0$, so we may assume that $n \geq 1$.

First, suppose that $0 \leq j \leq q_{n}-2$. Then $w_{j}$ is a factor of the word $z=s_{n}^{i} s_{n-1} s_{n}^{\prime \prime}$. If $n=1$, then $z$ clearly has a period $q_{n}$. If $n \geq 2$, then Equation (3) implies $z=s_{n}^{i+1} s_{n-1}^{\prime \prime}$, and we see that $z$ has a period $q_{n}$. Therefore also $w_{j}$ has a period $q_{n}$.

Next, suppose that $k q_{n} \leq j<(k+1) q_{n}$, where $1 \leq k \leq i-1$. This implies that $i \geq 2$. Then $w_{j}$ is a factor of the word

$$
z=s_{n}^{i-k} s_{n-1} s_{n}^{k} s_{n}^{\prime}
$$

We claim that $z$ is a prefix of the word $\left(s_{n}^{i-k} s_{n-1} s_{n}^{k-1}\right)^{3}$. Indeed, if $n=1$, verifying this is a straightforward computation. And if $n \geq 2$, the claim follows by an application of Equation (3). Hence $z$, and consequently also $w_{j}$, has a period $(i-1) q_{n}+q_{n-1}$.

Finally, suppose that $i q_{n} \leq j \leq i q_{n}+q_{n-1}-2$. This implies $n \geq 2$. Then the word $w_{j}$ is a factor of $z=s_{n-1} s_{n}^{i} s_{n-1}^{\prime \prime}$. By Equation (3), we can write $z=s_{n-1} s_{n}^{i-1} s_{n-1} s_{n}^{\prime \prime}$, and hence $z$, and also $w_{j}$, have a period $(i-1) q_{n}+q_{n-1}$.

Since $s_{n}^{i} s_{n-1}$ is a primitive word over a two-letter alphabet, it has at least two conjugates that are Lyndon words, and therefore unbordered. We have seen that $w_{j}$ is bordered in all other cases except possibly when $j=q_{n}-1$ and $j=$ $i q_{n}+q_{n-1}-1$, so that the last claim of the lemma holds. 
Lemma 7. A word $w$ is an unbordered factor of $\mathbf{x}$ if and only if $w=t_{-1}, w=t_{0}$, or $w$ is one of the two Lyndon words that are conjugates of $t_{m}$ for some $m \geq 1$.

Proof. According to Lemmas 5 and 6 , the claim holds if $|w|=\left|t_{m}\right|$ for some $m \geq-1$. Hence we may suppose that $|w| \neq\left|t_{m}\right|$ for all $m \geq-1$. We will show that $w$ is bordered.

First, observe that we have $|w|>\left|t_{d_{1}}\right|=d_{1}+1$ because $\left|t_{i}\right|=i+1$ for $i=$ $0,1, \ldots, d_{1}$. Furthermore, there exists an integer $n \geq 1$ such that either

$$
q_{n}<|w|<q_{n}+q_{n-1} \quad \text { or } \quad i q_{n}+q_{n-1}<|w|<(i+1) q_{n}+q_{n-1}
$$

for some $1 \leq i<d_{n+1}$. It follows that $w$ is a proper prefix of some factor of $\mathbf{x}$ of length $i q_{n}+q_{n-1}$ with $1 \leq i \leq d_{n+1}$ such that

$$
|w|>\max \left\{q_{n},(i-1) q_{n}+q_{n-1}\right\} .
$$

Denote this factor by $z$. Then $z$ is either a conjugate of $s_{n}^{i} s_{n-1}$, or the singular factor corresponding to $s_{n}^{i} s_{n-1}$. If $z$ is the singular factor, then $w$ is bordered because $z$ has a period $q_{n}$ and $|w|>q_{n}$. Hence we may suppose that $z$ is a conjugate of $t_{m}=s_{n}^{i} s_{n-1}$.

If $z$ is bordered, then according to Lemma $6, z$ has a period $q_{n}$ or a period $(i-1) q_{n}+q_{n-1}$. In either case, $z$ has a period strictly less than $|w|$, and so $w$ is bordered.

If $z$ is unbordered, Lemma 6 implies that either

$$
z=\sigma^{-1}\left(s_{n}^{i} s_{n-1}\right) \quad \text { or } \quad z=\sigma^{q_{n}-1}\left(s_{n}^{i} s_{n-1}\right) .
$$

Now we have two possibilities regarding as to whether $n=1$ or $n \geq 2$.

Suppose first that $n=1$. Then either $z=0\left(0^{d_{1}} 1\right)^{i}$ or $z=\left(10^{d_{1}}\right)^{i} 0$. In the first case, the inequality in (7) implies that $w=0\left(0^{d_{1}}\right)^{i-1} 0^{j}$ for some $j \geq 1$, so that $w$ is bordered. Similarly, in the second case we have $w=\left(10^{d_{1}}\right)^{i-1} 10^{j}$, where $1 \leq j \leq d_{1}$. If $i=1$, the word $w$ is a conjugate of $t_{j}$, a contradiction. Therefore, $i \geq 2$, and $w$ is bordered.

Suppose then that $n \geq 2$. Now, the word $w$ is a factor of either

$$
\sigma^{-2}\left(s_{n}^{i} s_{n-1}\right) \quad \text { or } \quad \sigma^{q_{n}-2}\left(s_{n}^{i} s_{n-1}\right) .
$$

Since we have already proved that $w$ is bordered if $w$ is a factor of a bordered word of length $i q_{n}+q_{n-1}$, we only have to show that both words in (9) are bordered. To do that, we only have to show that they are distinct from the words in (8). There are four cases to consider; one of them is

$$
\sigma^{-2}\left(s_{n}^{i} s_{n-1}\right)=\sigma^{q_{n}-1}\left(s_{n}^{i} s_{n-1}\right) .
$$

Since $s_{n}^{i} s_{n-1}$ is primitive, we get $i q_{n}+q_{n-1}-2=q_{n}-1$, which implies that $n \leq 1$, a contradiction. The remaining three cases are proved similarly; we omit details here. 
The next result by de Luca and De Luca appears in the proof of [7], Theorem 10. The original proof was obtained with a clever use of Duval extensions and a result of Mignosi and Zamboni [12]. Here we give a different, more constructive, albeit longer, proof.

Lemma 8 (de Luca, De Luca). The least period of a factor $w$ of $\mathbf{x}$ equals the length of a longest unbordered factor of $w$.

Proof. Let $u$ denote a longest unbordered factor of $w$. The claim clearly holds if $u$ is a letter, so we may assume that $|u| \geq 2$. Clearly, $p(w) \geq|u|$. To show that $w$ has a period $|u|$, it suffices to show that all factors of length $|u|$ of $w$ are conjugates of $u$.

To do that, suppose, contrary to what we want to show, that $w$ has a factor $z$ of length $|u|$ that is not a conjugate of $u$. Since the reversal of $w$ is also a factor of $\mathbf{x}$, we may, possibly by replacing $w$ by $w^{R}$, assume that $u$ occurs on the left of $z$ in $w$. Let $v$ denote a prefix of $w$ such that $z$ is a suffix of $v$ and $u$ is a factor of $v$.

Since $u$ is unbordered and $|u| \geq 2$, Lemma 7 implies that $u$ is a conjugate of $t_{m}=s_{n}^{i} s_{n-1}$ for some $n \geq 0$ and $1 \leq i \leq d_{n+1}$. Therefore, $z$ is the singular factor corresponding to $s_{n}^{i} s_{n-1}$. Hence, if $a$ denotes the last letter of $s_{n-1}$, then it follows from Lemma 5 that $z=\bar{a} s_{n}^{i} s_{n-1}^{\prime}$.

Next, denote $p=s_{n} s_{n+1}=s_{n}^{d_{n+1}+1} s_{n-1}$. Observe that, as a suffix of $s_{n+2} s_{n+1}$, the word $a p$ is a factor of $\mathbf{x}$.

Let us denote the longest common suffix of $a p$ and $v a$ by $y$. Since $z a$ is a suffix of both $p$ and $v a$, it is a suffix of $y$, as well. By Lemma 6, the word

$$
a p^{\prime}=\sigma^{-1}\left(s_{n}^{d_{n+1}+1} s_{n-1}\right)
$$

is unbordered. Since $\left|a p^{\prime}\right|=\left|s_{n}^{d_{n+1}+1} s_{n-1}\right|>|u|$, it then follows that $y$ is a proper suffix of $a p$ because otherwise $a p^{\prime}$ is a factor of $w$, contradicting the maximality of $|u|$.

Since $p$, and hence also $y$, has a period $q_{n}$, the word $u$ cannot be a factor of $y$ because $u$ is unbordered and $|u|=i q_{n}+q_{n-1}$. Consequently, $y$ is also a proper suffix of $v a$. This implies that $y$ is a left special factor of $\mathbf{x}$, and as such, a prefix of c. In particular, $s_{n}$ is a prefix of $y$. Now the primitivity of $s_{n}$ and the fact that $y$ is a suffix of $p=s_{n}^{d_{n+1}+1} s_{n-1}$ imply that we have $y=s_{n}^{j} s_{n-1}$ for some $i+1 \leq j \leq d_{n+1}+1$ (for the left inequality, note that $|y|>|u|=\left|s_{n}^{i} s_{n-1}\right|$.

We can rule out the possibility that $j=d_{n+1}+1$ because the word $s_{n}^{d_{n+1}+1} s_{n-1}$ is not a prefix of $\mathbf{c}$. Indeed, this is straightforward to verify for $n=0$ and $n=1$, and Theorem 1 handles the case when $n \geq 2$.

Now, we see that $\bar{a} y$ is a suffix of $p$. Since $y$ is a proper suffix of $v a$, the maximality of $y$ implies that $a y$ is a factor of $v a$. Therefore $a y^{\prime}$ is a factor of $v$, and hence of $w$. But $a y^{\prime}=a s_{n}^{j} s_{n-1}^{\prime}$ is unbordered by Lemma 6, and $\left|a y^{\prime}\right| \geq|z a|>|u|$, contradicting the maximality of $u$. The proof is complete.

The next result is the strongest result in this section, and it gives our desired formula for the period set of $\mathbf{x}$ as a corollary. 
Theorem 3. The fractional root of a factor of $\mathbf{x}$ is a conjugate of $t_{m}$ for some $m \geq-1$.

Proof. Let $w$ be a factor of $\mathbf{x}$. If $w$ is unbordered, then according to Lemma 7 , it is a conjugate of some $t_{m}$, where $m \geq-1$. If $w$ is bordered, Lemmas 8 and 7 imply that $p(w)=\left|t_{m}\right|$ for some $m \geq 0$. Consequently, the fractional root of $w$ is either a conjugate of $t_{m}$, or the singular factor $\bar{a} t_{m}^{\prime}$, where $a$ is the last letter of $t_{m}$. In the first case the claim holds, so may suppose that $\bar{a} t_{m}^{\prime}$ is the fractional root of $w$.

Since $p(w)<|w|$, it follows that $t_{m}^{\prime} \bar{a}$ is a factor of $\mathbf{x}$. By the definition of a singular factor, no other conjugates of $\bar{a} t_{m}^{\prime}$ except $\bar{a} t_{m}^{\prime}$ itself are factors of $\mathbf{x}$. Therefore, $\bar{a} t_{m}^{\prime}=t_{m}^{\prime} \bar{a}$. This implies that the fractional root of $w$ is actually the letter $\bar{a}$, and the claim follows.

Theorem 3 implies the following characterization of the period set of $\mathbf{x}$.

Corollary 3. The period set of $\mathbf{x}$ is the set

$$
\left\{\left|t_{m}\right|: m \geq-1\right\}=\{1\} \cup\left\{i q_{n}+q_{n-1}: n \geq 0, i=1, \ldots, d_{n+1}\right\} .
$$

The famous Fibonacci word is the characteristic sequence with slope $1 / \phi$, where $\phi=(1+\sqrt{5}) / 2$ denotes the golden ratio. As a special case of Corollary 3, we obtain the next result, which was first proved in [14].

Corollary 4. The least period of a factor of the Fibonacci word is a Fibonacci number.

\section{Applications}

In this section we give four applications of our results in the previous section. The first application by Harju and Nowotka [9] is a direct corollary of Lemma 7.

Corollary 5. Unbordered words that are factors of Sturmian words are Lyndon words.

The next characterization of finite Sturmian words is by de Luca and De Luca [7], Theorem 10 .

Corollary 6. A finite word is a factor of a Sturmian word if and only if its fractional root is a conjugate of a standard word.

Proof. Let a finite word $w$ be a factor of a Sturmian word, say a factor of $\mathbf{x}$ using the notation from the last section. Then by Theorem 3 the fractional root of $w$ is a conjugate of $t_{m}$ for some $m \geq-1$, and $t_{m}$ is a standard word.

Conversely, suppose $w=u^{\tau}$, where $u$ is a conjugate of a standard word, say $s_{n}$, and $\tau \geq 1$ is rational. Then $w$ is a factor of $s_{n}^{a+2}$, where $a=\lfloor\tau\rfloor$, which clearly is a prefix of a characteristic word. 
Our last two corollaries below use a well-known theorem by Fine and Wilf [8], which states that if two words $x^{n}$ and $y^{m}$ have a common prefix of length $|x|+$ $|y|-\operatorname{gcd}(|x|,|y|)$, then both of them have a period $\operatorname{gcd}(|x|,|y|)$.

Here is one more application of Theorem 3, see also Damanik and Lenz [6].

Corollary 7. If a square uu is a factor of $\mathbf{x}$ and $u$ is primitive, then $u$ is a conjugate of $t_{m}$ for some $m \geq 0$.

Proof. Let $v$ denote the fractional root of $u$, which by Theorem 3 is a conjugate of $t_{m}$ for some $m \geq-1$. The word 11 does not occur in $\mathbf{x}$, so that $m \geq 0$. Then $u u=v^{\tau}$ for some rational $\tau \geq 2$, and we have $|u|+|v| \leq|u u|$. By the theorem of Fine and Wilf, $u u$ has a period $\operatorname{gcd}(|u|,|v|)$. Since $v$ is the fractional root of $u u$, this implies that $|v|=\operatorname{gcd}(|u|,|v|)$, and hence $|v|$ divides $|u|$. Since $u$ is primitive, it follows that $u=v$.

Cummings et al. [5] gave two proofs showing that, for $n \geq 2$, the least period of the finite Fibonacci word $f_{n}$ is $f_{n-1}{ }^{2}$. As our last result of this chapter, we generalize the result of Cummings et al. to standard words. Let us use the notation from the previous section, that is, $s_{n}$ is a standard word and $s_{n}=s_{n-1}^{d_{n}} s_{n-2}$.

Corollary 8. If $n \geq 2$, then the least period of $s_{n}$ equals $q_{n-1}$.

Proof. Since $s_{n}=s_{n-1}^{d_{n}} s_{n-2}$ and $s_{n-2}$ is a prefix of $s_{n-1}$, we see that $q_{n-1}$ is a period of $s_{n}$. Hence we only need to show that $q_{n-1}$ is the least period. To do that, suppose the contrary: we have $p\left(s_{n}\right)<q_{n-1}$.

First, suppose that $d_{n} \geq 2$. Since $s_{n}$ has periods $q_{n-1}$ and $p\left(s_{n}\right)$, and

$$
q_{n-1}+p\left(s_{n}\right)<2 q_{n-1}<q_{n},
$$

it follows from the theorem of Fine and Wilf that $p\left(s_{n}\right)$ is a proper divisor of $q_{n-1}$. Since $s_{n-1}$ is a prefix of $s_{n}$, this implies that $s_{n-1}$ is not primitive, a contradiction.

Second, suppose that $d_{n}=1$. If $p\left(s_{n}\right) \leq q_{n-2}$, we derive a contradiction as above. Therefore we may assume that $p\left(s_{n}\right)>q_{n-2}$. Now, Theorem 3 implies that $p\left(s_{n}\right)=i q_{n-2}+q_{n-3}$ with $1 \leq i<d_{n-1}$. Then the word $s_{n-2}^{i} s_{n-3} s_{n-2}$ is a prefix of $s_{n}$. But since $s_{n-1}=s_{n-2}^{d_{n-1}} s_{n-3}$ is also a prefix of $s_{n}$, we obtain $s_{n-3} s_{n-2}=$ $s_{n-2} s_{n-3}$, which is absurd by Equation (2). This contradiction completes the proof.

Acknowledgements. The second author is grateful to Alessandro De Luca for pointing out the result stated in Lemma 8. A sincere thank you also to Gwénaël Richomme for pointing out a deficiency in the first version of Lemma 2 and also for other useful comments that helped to clarify the presentation. Finally, thank you to the referees for their remarks.

\footnotetext{
${ }^{2}$ To be precise, Cummings et al. showed that the longest border of $f_{n}$ is $f_{n-2}$, but these two claims are equivalent.
} 


\section{REFERENCES}

[1] J.-P. Allouche and J. Shallit, The ubiquitous Prouhet-Thue-Morse sequence, in Sequences and Their Applications: Proceedings of SETA'98. Springer Series in Discrete Mathematics and Theoretical Computer Science, C. Ding, T. Helleseth and H. Niederreiter, Eds., Springer-Verlag, London (1999) 1-16.

[2] J. Berstel, On the index of Sturmian words. In Jewels are forever. Springer, Berlin (1999) $287-294$.

[3] W.-T. Cao and Z.-Y. Wen, Some properties of the factors of Sturmian sequences. Theor. Comput. Sci. 304 (2003) 365-385.

[4] C. Choffrut and J. Karhumäki, Combinatorics on words. In A. Salomaa and G. Rozenberg, Eds., Handbook of Formal Languages, volume 1. Springer, Berlin (1997) 329-438.

[5] L.J. Cummings, D.W. Moore and J. Karhumäki, Borders of Fibonacci strings. J. Comb. Math. Comb. Comput. 20 (1996) 81-87.

[6] D. Damanik and D. Lenz, Powers in Sturmian sequences. Eur. J. Combin. 24 (2003) 377390.

[7] A. de Luca and A. De Luca, Some characterizations of finite Sturmian words. Theor. Comput. Sci. 356 (2006) 118-125.

[8] N.J. Fine and H.S. Wilf, Uniqueness theorems for periodic functions. Proc. Amer. Math. Soc. 16 (1965) 109-114.

[9] T. Harju and D. Nowotka, Minimal Duval extensions. Int. J. Found. Comput. Sci. 15 (2004) 349-354.

[10] M. Lothaire, Combinatorics on Words. Cambridge University Press, Cambridge (1997).

[11] M. Lothaire, Algebraic Combinatorics on Words, Encyclopedia of Mathematics and its Applications, Vol. 90. Cambridge University Press, Cambridge (2002).

[12] F. Mignosi and L.Q. Zamboni, A note on a conjecture of Duval and Sturmian words. RAIROTheor. Inf. Appl. 36 (2002) 1-3.

[13] M. Mohammad-Noori and J.D. Currie, Dejean's conjecture and Sturmian words. Eur. J. Combin. 28 (2007) 876-890.

[14] K. Saari, Periods of factors of the Fibonacci word. in Proceedings of the Sixth International Conference on Words (WORDS'07). Institut de Mathématiques de Luminy (2007) 273-279.

Communicated by J. Karhumäki.

Received November 28, 2007. Accepted February 6, 2008. 